\title{
Soluble Markers of Antibody Secreting Cell Function as Predictors of Infection Risk in Rheumatoid Arthritis
}

\author{
Maria J. Gutierrez ${ }^{(D)}{ }^{1}$ Stephen V. Desiderio, ${ }^{2}$ Nae-Yuh Wang, ${ }^{3,4,5}$ Erika Darrah, $^{6}$ \\ Laura Cappelli, ${ }^{6}$ Gustavo Nino, ${ }^{7}$ Michelle Jones $\mathbb{D}^{6},{ }^{6}$ and Clifton O. Bingham III ${ }^{6,8}$ \\ ${ }^{1}$ Division of Pediatric Allergy and Immunology, Johns Hopkins University School of Medicine, Baltimore, MD, USA \\ ${ }^{2}$ Department of Molecular Biology and Genetics, Johns Hopkins University School of Medicine, Baltimore, MD, USA \\ ${ }^{3}$ Department of Medicine, Division of General Internal Medicine, Johns Hopkins University School of Medicine, Baltimore, MD, USA \\ ${ }^{4}$ Department of Biostatistics, Johns Hopkins Bloomberg School of Public Health, Baltimore, MD, USA \\ ${ }^{5}$ Department of Epidemiology, Johns Hopkins Bloomberg School of Public Health, Baltimore, MD, USA \\ ${ }^{6}$ Division of Rheumatology, Johns Hopkins University School of Medicine, Baltimore, MD, USA \\ ${ }^{7}$ Division of Pulmonary and Sleep Medicine, Children's National Medical Center, Washington, DC, USA \\ ${ }^{8}$ Division of Allergy and Clinical Immunology, Johns Hopkins University School of Medicine, Baltimore, MD, USA
}

Correspondence should be addressed to Maria J. Gutierrez; mgutie10@jhmi.edu

Received 2 February 2019; Accepted 25 March 2019; Published 28 April 2019

Guest Editor: Di Yu

Copyright (c) 2019 Maria J. Gutierrez et al. This is an open access article distributed under the Creative Commons Attribution License, which permits unrestricted use, distribution, and reproduction in any medium, provided the original work is properly cited.

Background. Rheumatoid arthritis (RA) is a systemic autoimmune disease associated with immune dysregulation and increased risk of infections. The presence of autoantibodies and immunoglobulin abnormalities indicates B-cell and antibody-secreting cell (ASC) dysfunction. We hypothesize that soluble factors associated with B-cell and ASC activity are decreased in RA patients and that this is linked to higher susceptibility to infections. Methods. Using the Johns Hopkins Arthritis Cohort and Biorepository, we contrasted serum protein levels of soluble factors involved in B-cell activation (CD40, CD40L) and B-cell/ASC homing (CXCL10, CXCL11, and CXCL13) or survival (BAFF, APRIL, TACI, and BCMA) in 10 healthy subjects and 23 adult RA patients (aged 24-65 years). We subdivided RA patients into those with $(n=17)$ and those without infections $(n=6)$ within a 2 -year period. In order to reduce the effect of RA treatment, we only included patients receiving methotrexate monotherapy or no RA treatments at baseline. Soluble serum protein levels of B-cell/ASC factors were quantified by multiplex immunoassays. Results. We identified that (1) serum levels of soluble BCMA, APRIL, CD40, and CD40L were significantly decreased in RA patients relative to healthy individuals; (2) serum soluble BCMA, predominantly released by ASC, correlated with serum concentrations of class-switched immunoglobulins, IgG and IgA; and (3) RA patients with a history of infections had significantly lower soluble BCMA levels compared with healthy donors and with RA patients without infections. Conclusions. Our study using soluble factors linked to B-cell/ASC activation and survival suggests that there is a paucity of ASC in a subset of RA patients and that this may be linked to altered antibody production and increased risk of infections. Further delineating the link between ASC and infection susceptibility in RA may optimize disease management and provide novel insights into disease pathogenesis that are susceptible to intervention.

\section{Introduction}

Rheumatoid arthritis (RA) is the most common systemic autoimmune disease, affecting approximately $0.5-1 \%$ of adults worldwide [1]. It is characterized by synovial inflammation, autoantibody production, and systemic features
$[1,2]$. Although inflammatory manifestations predominate in RA, some affected individuals also have an intrinsically increased susceptibility to infections [2-4]. Indeed, a higher incidence of bronchitis and pneumonia, sometimes preceding joint disease $[4,5]$, higher frequency of tuberculosis (TB) [6], and increased mortality due to infections $[3,7]$ 
are described before steroids and immunomodulators were used. This is particularly concerning since RA patients usually require medications that further suppress pathways integral to immune function, and as a consequence, infections remain a leading complication in RA [8].

Although the association between RA and increased susceptibility to infections has long been recognized [4], the causes underlying this phenomenon remain poorly defined. Some patients with RA have hypogammaglobulinemia [9-13] suggesting that impaired production of antibodies could accompany the disease. Antibodies against infections are produced by antibody-secreting cells (ASC) including plasmablasts and plasma cells $[14,15]$. However, autoimmunity can also result from anomalous production of autoantibodies by self-reactive ASC [2, 16, 17]. Therefore, ASC defects may play a dual role in the production of pathogenic autoantibodies as well as in deficient responses to infections in RA $[14,16,17]$. Additional support for this notion is provided by the study of primary antibody immunodeficiencies, in which RA and other autoantibody-associated diseases are more common [18-20].

To produce antibodies, B-cells must first mature into ASC, which may be short-lived effectors in early antibody responses or prolonged lifespan plasma cells that produce long-lasting, class-switched, highly specific antibodies (e.g., immunoglobulins A, G) $[14,15,21]$. Short-lived ASC are produced upon direct antigen stimulation or during early T-cell-dependent responses [14]. Long-lived ASC are generated in a complex process triggered by cell-to-cell interactions with follicular T-helper cells via CD40 and CD40 ligands (CD40L), followed by extensive proliferation of activated B-cells to generate germinal centers [14, 22, 23]. During this process, class-switch recombination (CSR) in B-cells occurs to produce antibody isotypes (e.g., IgA, IgG, and $\operatorname{IgE})[14,15,22]$. Germinal center reactions under $\mathrm{T}$-cells help also cause the proliferation and selection of activated B-cells to produce highly specific antibodies against encountered antigens $[14,15]$. After these processes, longlived ASC producing antibodies of high affinity and variable isotypes are generated. During ASC generation, B-cells and ASC migrate in processes controlled by B-cell trafficking molecules such as CXCL9, CXCL10, and CXCL11 that, among other functions, home cells to inflamed tissues or CXCL13 that directs B-cell/ASC in germinal centers [14, 21]. Most newly differentiated B-cells undergo apoptosis unless survival signals are delivered by two members of the TNF receptor superfamily, BAFF (B-cell-activating factor) and APRIL (a proliferation-inducing ligand) via binding to their receptors TACI (transmembrane activator and calcium modulator and cyclophilin ligand interactor) $[14,24]$ and BAFF-R (BAFF receptor) [24, 25]. Another BAFF/APRIL receptor, $\mathrm{BCMA}$ (B-cell maturation antigen), is predominantly expressed by ASC and is essential to ensuring the prolonged lifespan of plasma cells $[26,27]$.

Although humoral immune responses have been extensively studied in RA, studies often focus on the role of ASC in the production of autoantibodies [1, 2, 28, 29]. It remains unclear whether ASC abnormalities in RA also alter normal protective antibody responses against infections or immunizations. The study of human ASC in rheumatologic diseases is limited by the fact that most, especially long-lived, plasma cells reside in the bone marrow and are usually not accessible in peripheral blood [14, 15, 21]. Thus, our knowledge about the mechanisms regulating the development and function of ASC in individuals with $\mathrm{RA}$ is incomplete.

The goal of this study was to study whether individuals with RA exhibit abnormalities in serum levels of soluble factors linked to critical steps for ASC generation, including those for B-cell activation and B-cell/ASC survival and trafficking. Specifically, we aimed to characterize differences in such factors between patients with RA and healthy individuals. We also sought to examine associations between some of those factors and clinical outcomes linked to ASC function, such as antibody production and infections.

\section{Methods}

2.1. Subjects. We conducted a retrospective evaluation of clinical data from patients included in the Johns Hopkins Arthritis Cohort (JHAC) [30]. The JHAC has detailed longitudinal clinical information in over 730 active RA patients followed during clinical visits and a biorepository containing serum samples from approximately $80 \%$ of enrolled subjects. For the present study, our patient population was drawn from individual RA subjects enrolled in the JHAC since $2012(n=277)$ with serum samples collected on the same day as a clinical assessment. We included all patients aged 18 to 65 years old, with a provisional RA diagnosis (physician assessment and use of disease-modifying antirheumatic drugs (DMARD)) or meeting American College of Rheumatology 2010 criteria for RA [31], receiving monotherapy with methotrexate (MTX) or taking no immunomodulators at serum sample collection. Serum samples were part of the JHAC biorepository. Demographic information and stored serum from 10 healthy control volunteers were also used. All samples and data were collected after informed written consent approved by the Johns Hopkins Institutional Review Board.

2.2. Clinical Data. Basic demographic and clinical characteristics (sex, age of onset, autoantibody status, disease duration, disease activity, medication use, and MTX dose) at the time of the baseline visit (when serum sample was collected) were extracted from the JHAC clinical database. Seropositive arthritis was defined as RA associated with positive rheumatoid factor (RF) or anticitrullinated protein antibodies (ACPA) as previously described. RA activity was determined using the Clinical Disease Activity Index (CDAI), classified as remission (CDAI $\leq 2.8)$, low disease activity $(\mathrm{CDAI}>2.8$ and $\leq 10)$, moderate disease activity (CDAI $>10$ and $\leq 22$ ), and high disease activity $(\mathrm{CDAI}>22)$ as previously defined [32]. Disease onset was defined as the age at which RA symptoms started, and disease duration was established as the time since the onset of RA symptoms to the date of sample collection in years. Infectious events were self-reported using a patient questionnaire inquiring about joint/bursa, cellulitis/skin, sinusitis, diverticulitis, sepsis, pneumonia, 


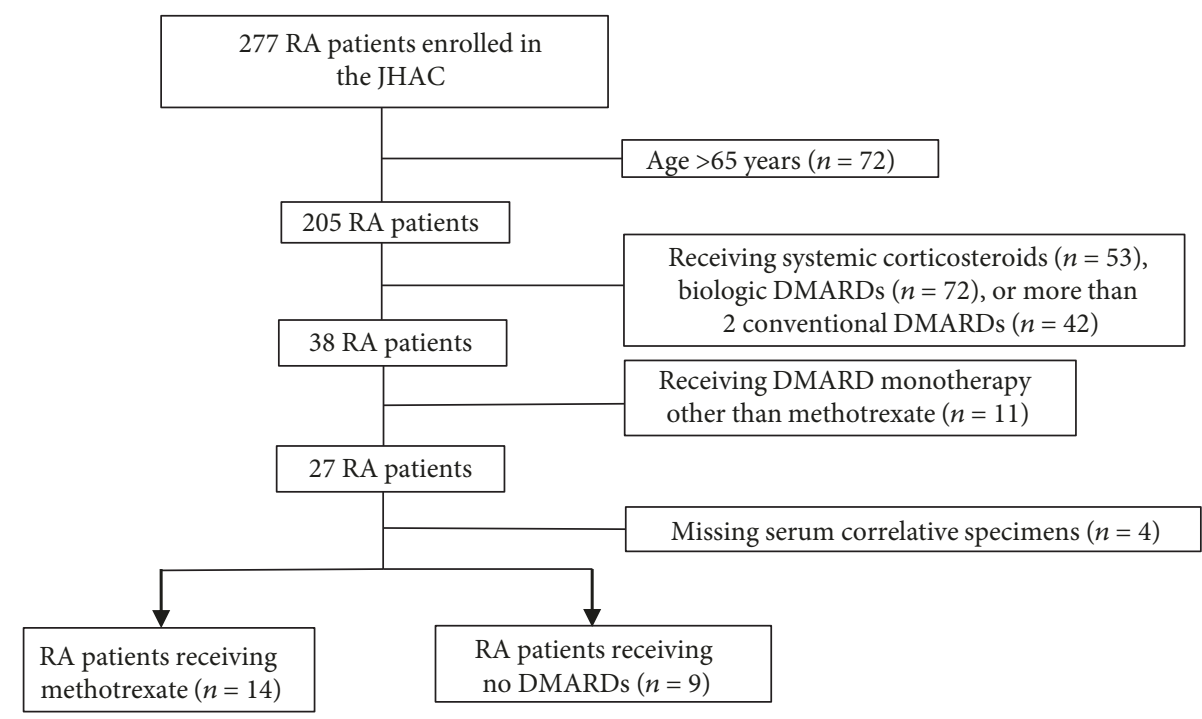

FIgURE 1: Flow diagram shows selection of patients included in our study. A total of 23 RA patients from the JHAC were included in the final analysis. Abbreviations: RA=rheumatoid arthritis; JHAC=Johns Hopkins Arthritis Cohort; DMARDs=disease-modifying antirheumatic drugs.

bronchitis, gastroenteritis, meningitis/encephalitis, upper respiratory infections (URI), an urinary tract infection (UTI) at every visit. Infections not included in these categories were self-reported under "other infection." Information on whether hospitalization and/or parenteral antibiotics associated with these infectious events was also recorded.

\subsection{Measurement of B-Cell/ASC-Soluble Serum Factors and} Immunoglobulins. Measurements of soluble factors involved in B-cell/ASC activation, survival, and homing as well as serum immunoglobulin concentrations were performed on serum samples from the JHAC biorepository. We quantified serum protein levels of soluble factors involved in B-cell activation (CD40, CD40L) and B-cell/ASC survival (APRIL, BAFF, sTACI, and sBCMA) or homing (CXCL10, CXCL11, and CXCL13) using a magnetic bead-based multiplex immunoassay (R\&D Human Magnetic Luminex Assay). All samples, including healthy control samples, were processed simultaneously. Serum concentrations of immunoglobulins A (IgA), G (IgG), and M (IgM) in RA patients were assessed by nephelometry at the Johns Hopkins Hospital Clinical Immunology laboratory.

2.4. Statistical Analyses. Data were analyzed using the software STATA version 14 (StataCorp. Stata Statistical Software: Release 14. College Station, TX. 2015), R studio version 1.10.153 (RStudio Team (2018). RStudio: Integrated Development for R. RStudio Inc., Boston, MA), and Minitab Student version 14 (Minitab 14 Statistical Software (2018). Minitab Inc., State College, PA). Basic clinical characteristics of all RA patients were extracted from the JHAC clinical database. Age, age of onset, disease duration, disease activity (Clinical Disease Activity Index score (CDAI) score), and MTX dose are summarized as medians and interquartile ranges (IQR) as distributions were non-Gaussian and were compared using the Wilcoxon rank-sum test. Differences in sex, ethnicity, and autoantibody status between RA subgroups were contrasted using Fisher's exact test. Differences in B-cell/ASC activation, survival, and homing factors between RA patients and healthy controls (HC) were examined using a Wilcoxon rank-sum test, and Spearman's correlations were used to evaluate the relationship between sBCMA, serum immunoglobulins (IgA, IgG, and IgM) and selected B-cell/ASC activation, and survival factors (APRIL, CD40, and CD40L). Differences between HC and the two RA subgroups (RA with and without infections) were compared using nonparametric methods (Kruskal-Wallis tests to assess differences among groups and Wilcoxon rank-sum tests for pair-wise comparisons) or a chi-square test as indicated. Finally, since controls and RA patients were not age-matched, we built linear regression models to evaluate whether differences in B-cell/ASC activation, homing, and survival factors (using log-transformed values) between healthy controls and RA patients persisted after adjusting by age at baseline.

\section{Results}

3.1. Study Population. Clinical data from 277 patients enrolled in the JHAC database and biorepository were screened for eligibility. There were 205 subjects at serum sample collection who met age eligibility criteria (Figure 1). We excluded 53 patients receiving systemic corticosteroids, 72 receiving biologic DMARDs, and 42 treated with more than one conventional DMARD. We also removed patients taking sulfasalazine, as it can be associated with hypogammaglobulinemia or monotherapy with a conventional DMARD other than methotrexate (MTX). After excluding 4 eligible subjects with insufficient serum specimens, we included 23 RA patients in the final analysis (Figure 1).

The main demographic and clinical characteristics of subjects included are displayed in Table 1. The median age of RA patients was 55 years (IQR 16, range 24-64 years), with 
TABLE 1: Basic demographic and clinical characteristics of RA patients and RA subgroups.

\begin{tabular}{|c|c|c|c|c|}
\hline & $\begin{array}{l}\text { Healthy donors } \\
\qquad N=10\end{array}$ & $\begin{array}{l}\text { RA patients without infections } \\
\qquad N=17\end{array}$ & $\begin{array}{l}\text { RA patients with infections } \\
\qquad N=6\end{array}$ & $p$ value \\
\hline \multicolumn{5}{|l|}{ Gender } \\
\hline Male/female & $1 / 9$ & $4 / 13$ & $0 / 6$ & 0.54 \\
\hline \multicolumn{5}{|l|}{ Ethnicity, $n(\%)$} \\
\hline White/Caucasian & 6 & $13(76.5)$ & $3(50)$ & \\
\hline Non-white & 4 & $4(23.5)$ & $3(50)$ & 0.33 \\
\hline \multicolumn{5}{|l|}{ Age in years } \\
\hline Median (IQR) & $41(26)$ & $54(15)$ & $55.5(9)$ & 0.09 \\
\hline \multicolumn{5}{|l|}{ Disease duration in years } \\
\hline Median (IQR) & & $5(6)$ & $10.5(11)$ & 0.32 \\
\hline \multicolumn{5}{|l|}{ Age of onset } \\
\hline Median (IQR) & & $39(25)$ & $41.5(20)$ & 0.97 \\
\hline \multicolumn{5}{|l|}{ Autoantibody status } \\
\hline Seropositive/seronegative RA & & $14 / 3$ & $4 / 2$ & 0.57 \\
\hline \multicolumn{5}{|l|}{ Disease activity (CDAI score) } \\
\hline Median (IQR) [range] & & $2.95(10.2)[0-37]$ & $8.5(4.0)[0-22]$ & 0.34 \\
\hline Number of patients & & 16 & 5 & \\
\hline \multicolumn{5}{|l|}{$R A$ medication category } \\
\hline MTX-treated/no DMARD & & $10 / 7$ & $4 / 2$ & 1.0 \\
\hline \multicolumn{5}{|l|}{ Methotrexate dose (mg/week) } \\
\hline Median (IQR) & & $17.5(10)$ & $22.5(2.5)$ & 0.30 \\
\hline Number of patients & & 9 & 3 & \\
\hline
\end{tabular}

Abbreviations: RA = rheumatoid arthritis; IQR = interquartile range; CDAI score = Clinical Disease Activity Index score.

19 females (83\%) and 4 males (17\%). Patients in our sample were predominantly Caucasian $(n=16,70 \%)$. The median age of disease onset was 39 years (IQR 26), and median disease duration was 7 years (IQR 11). In regards to disease activity, patients had a median CDAI of 3.9 (IQR 9.5). Nine patients were in remission, six had low disease activity, and six had moderate $(n=4)$ or high disease activity $(n=2)$. There were two patients with no disease activity scores available $(n=2)$. We identified 14 patients treated with MTX only (61\%) and 9 patients receiving no DMARDs (39\%) at the time of serum sample collection. Healthy donors for this study included 10 healthy volunteers $(n=10)$ of both sexes aged 21 to 62 years (median 41 years, IQR 23) without history of significant medical conditions. No differences in age were detected among the three groups $(p=0.09)$; however, RA patients as a group were older when compared with healthy subjects $(p=0.03)$.

3.2. B-Cell Activation and Survival Factors Were Significantly Decreased in RA Patients Compared with Healthy Individuals. In order to examine differences in soluble factors released during ASC generation in RA patients, we evaluated serum levels of soluble factors involved in B-cell/ASC activation, survival, and homing in RA subjects $(n=23)$ and healthy individuals $(n=10)$. We identified significantly reduced levels of APRIL (median levels $\mathrm{RA}=1,807.8 \mathrm{pg} / \mathrm{ml}$ vs. controls $=2,544.9 \mathrm{pg} / \mathrm{ml}, p=0.009$ ) and sBCMA (median levels $\mathrm{RA}=16,535.2 \mathrm{pg} / \mathrm{ml}$ vs. controls $=20,487.8 \mathrm{pg} / \mathrm{ml}$, $p=0.008$ ), the ligand-receptor duo responsible for maintaining ASC survival, in the serum RA subjects relative to controls. We also found reduced serum levels of soluble CD40 (median levels $\mathrm{RA}=482.3 \mathrm{pg} / \mathrm{ml}$ vs. controls = $608.3 \mathrm{pg} / \mathrm{ml}, p=0.02$ ) and CD40L (median levels $\mathrm{RA}=$ $4,446.3 \mathrm{pg} / \mathrm{ml}$ vs. controls $=12,450.5 \mathrm{pg} / \mathrm{ml}, \quad p<0.0001)$. Differences in sBCMA, CD40 and CD40L, and APRIL persisted after adjusting by age (Figure S1). Serum levels of soluble BAFF were slightly decreased in RA patients but were nonsignificantly different from healthy subjects in a bivariate analysis (median levels $\mathrm{RA}=913.9 \mathrm{pg} / \mathrm{ml}$ vs. controls $=1128.0 \mathrm{pg} / \mathrm{ml}, p=0.21$ ) or after adjusting by age $(p=0.52)$ (Figure S1). In contrast with other survival factors, the soluble receptor TACI was increased in RA. Differences with healthy donors failed to achieve statistical significance in an unadjusted analysis (median levels $\mathrm{RA}=$ $26.56 \mathrm{pg} / \mathrm{ml}$ vs. controls $=16.9 \mathrm{pg} / \mathrm{ml}, p=0.13$ ) and after adjusting by age $(p=0.42)$ (Figure S1). There were nonsignificant increases in CXCL13 (median levels RA = $68.34 \mathrm{pg} / \mathrm{ml}$ vs. controls $=51.89 \mathrm{pg} / \mathrm{ml}, p=0.24)$, in CXCL10 (median levels $\mathrm{RA}=34.73 \mathrm{pg} / \mathrm{ml}$ vs. controls $=24.09 \mathrm{pg} / \mathrm{ml}$, $p=0.07$ ), and in CXCL11 (median levels $\mathrm{RA}=47.30 \mathrm{pg} / \mathrm{ml}$ vs. controls $=61.90 \mathrm{pg} / \mathrm{ml}, p=0.07)$ in the unadjusted comparison (Figure 2). However, the small increases seen in CXCL10 and CXCL13 in RA patients became statistically significant when adjusting by age (Figure S1). 

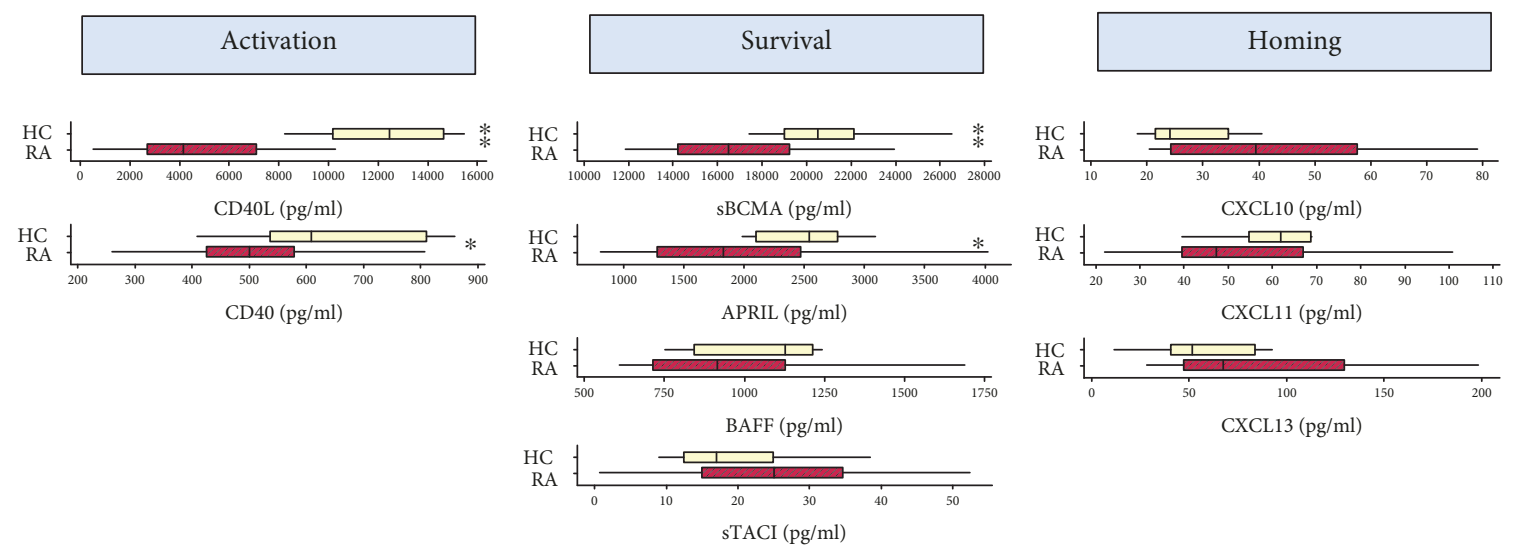

FIGURE 2: Box plots comparing serum B-cell factor protein levels in RA patients and healthy controls. Levels of each protein are indicated for healthy controls (HC, top yellow box; $n=10$ ) and RA patients (RA, bottom red box; $n=23$ ). The median value is indicated with a vertical line, with the box comprising the IQR, and whiskers representing the maximum and minimum values within the fences $( \pm 1.5 \mathrm{IQR}) .{ }^{* *} p<0.01$ and ${ }^{*} p<0.05$. Serum levels of B-cell activation factors (CD40, CD40L) and B-cell/ASC survival elements (sBCMA, APRIL) were significantly decreased in RA patients compared with healthy controls.

\subsection{Serum Soluble BCMA Protein Concentrations Had a} Positive Correlation with Serum Levels of Class-Switched Immunoglobulins in RA Patients. Because of CD40/CD40L interactions, APRIL and BCMA mediate critical steps in ASC generation and survival; we examined correlations between serum levels of the above factors and of classswitched immunoglobulins, IgG and IgA, as well as IgM. Median IgA, IgG, and IgM for RA patients were $207 \mathrm{mg} / \mathrm{dl}$ (range $4.9-472 \mathrm{mg} / \mathrm{dl}$ ), $1110 \mathrm{mg} / \mathrm{dl}$ (range $599-2290 \mathrm{mg} / \mathrm{dl}$ ), and $112 \mathrm{mg} / \mathrm{dl}$ (range $37-284 \mathrm{mg} / \mathrm{dl}$ ), respectively. Only serum sBCMA showed significant correlations with serum concentrations of class-switched immunoglobulins IgG (Spearman's rho $=0.6, p<0.01$ ) (Figure $3(\mathrm{~b})$ ) and IgA (Spearman's rho $=0.6, p<0.001$ ) (Figure 3(c)). There was also a strong correlation between serum IgA and IgG levels $($ rho $=0.8, p<0.001)$. Conversely, there were no significant correlations between $\mathrm{sBCMA}$ and IgM (Spearman's rho $=$ $0.0, p=0.9$ (Figure 3(d)) which is produced before classswitching. Finally, to a lesser degree, moderate correlations were found between sBCMA, CD40, and CD40L and between APRIL, CD40, and CD40L (Figure 3). Of note, there were three RA patients with mild IgG hypogammaglobuline$\mathrm{mia}(\operatorname{IgG}<640 \mathrm{mg} / \mathrm{dl})$ [33] and one subject with undetectable IgA serum levels $(\operatorname{IgA}<5 \mathrm{mg} / \mathrm{dl}$ ). These four subjects had sBCMA measurements in the lowest quartile for RA patients and below the minimum serum sBCMA level found in normal subjects. There were also two subjects with elevated IgG and IgA levels defined as serum concentrations above $1349 \mathrm{mg} / \mathrm{dl}$ [33] and $312 \mathrm{mg} / \mathrm{dl}$ [33], respectively, in spite of having sBCMA levels below the lowest level found in healthy donors $(17,420 \mathrm{pg} / \mathrm{ml})$. These two patients were characterized by having seropositive disease with high titers of ACPA antibodies (>200 IU).

3.4. Patients with RA and Infections Had Lower Serum sBCMA Levels Compared with RA Subjects without Infectious Events and Healthy Subjects. To investigate the clinical relevance of decreased serum sBCMA and its correlation with serum IgA and IgG levels, we examined the relationship between sBCMA and infections among RA patients. We subdivided the RA group into patients with a history of infections $(n=6)$ and those without infections $(n=17)$ within 2 years after collection of baseline serum samples. There were 10 infectious events reported during the follow-up period in six patients: 4 sinopulmonary infections, 2 influenza infections, 3 soft-tissue/mucosal infections, and 1 herpes zoster infection. We compared median serum sBCMA levels among the three groups (RA patients with infections $(n=6)$, RA patients without infections $(n=17)$, and healthy individuals $(n=10))$. The median serum sBCMA level was $14,669.7 \mathrm{pg} / \mathrm{ml}$ (IQR $2553 \mathrm{pg} / \mathrm{ml}$ ) in the RA subgroup with infections, $17,979.8 \mathrm{pg} / \mathrm{ml}$ (IQR $5777.3 \mathrm{pg} / \mathrm{ml}$ ) in the uninfected RA subgroup, and 20,487.8 (IQR $2561.3 \mathrm{pg} / \mathrm{ml}$ ) in healthy individuals, with statistically significant difference $(p=0.004)$ among these subgroups. Pair-wise comparisons revealed that RA patients with infections had significantly lower serum sBCMA levels compared with RA patients without infections $(p<0.05)$ and with healthy controls $(p<0.01)$ (Figure 4$)$. The levels of serum sBCMA in uninfected RA patients compared with healthy controls were not significantly different $(p=0.063)$. To examine whether this pattern persisted in the absence of methotrexate, we conducted a similar analysis in untreated patients (Figure S2) and continued to observe significantly decreased sBCMA levels in RA patients with infections $(n=2)$ compared with healthy controls $(n=10)(p=0.03)$. Serum sBCMA levels also were lower in patients with infections than in those without infections $(n=7)$, although this difference was not significant $(p=0.07)$.

3.5. Serum Immunoglobulins Were Not Associated with Infection Events in RA Patients. As serum immunoglobulin is clinically used to evaluate the production of antibodies $[33,34]$, we evaluated whether serum levels of $\operatorname{IgG}, \operatorname{IgA}$, and IgM predicted infectious events in RA patients. We compared serum concentrations of IgG, IgA, and IgM between the two patient groups (RA patients with infections $(n=6)$, RA patients without infections $(n=17))$ and evaluated the 


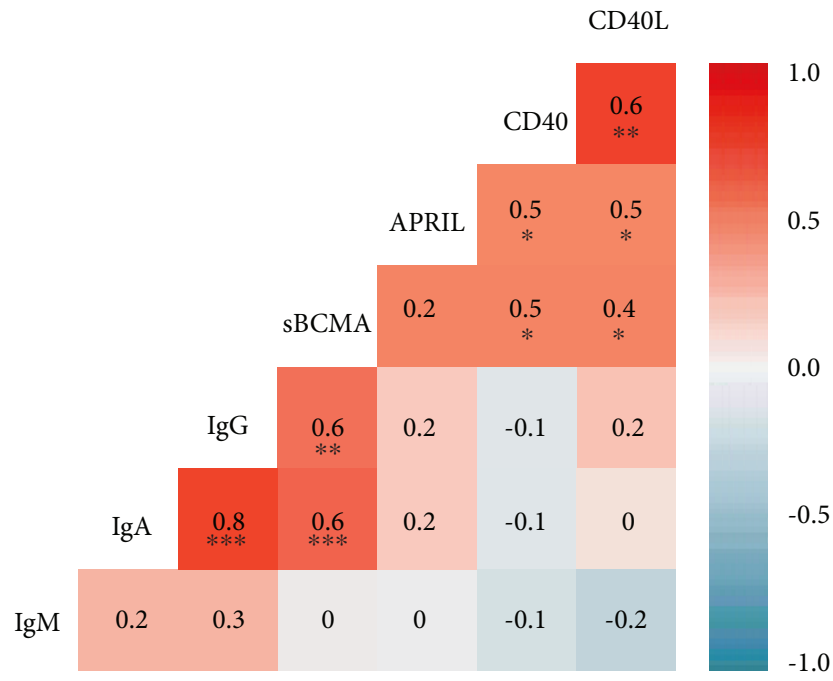

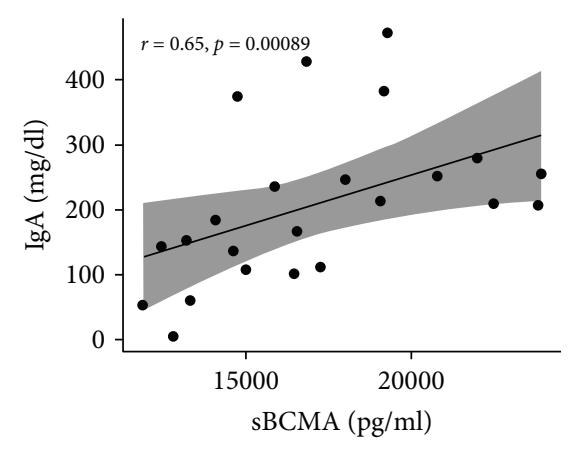

(b)

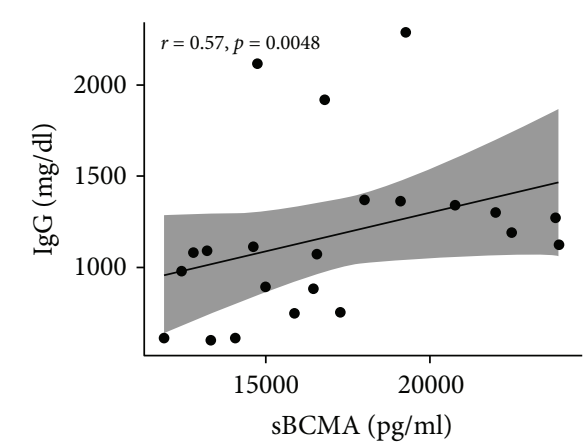

(c)

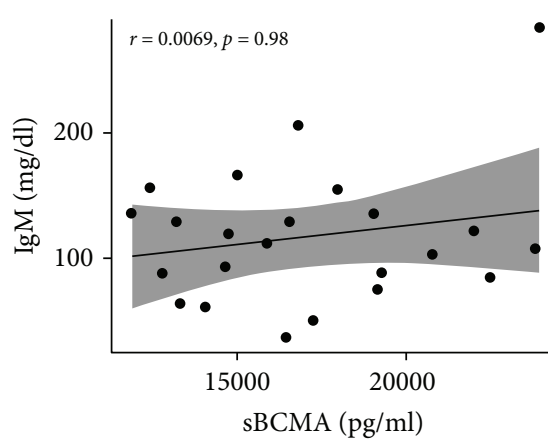

(d)

FIGURE 3: Correlation of B-cell factors and immunoglobulins in RA patients. (a) Spearman correlation matrix of soluble B-cell factors and Ig levels in RA. (b-d) Scatter plots demonstrating individual correlations between sBCMA and IgA (b), IgG (c), and IgM (d). Serum sBCMA (but not CD40, CD40L, or APRIL) had a positive correlation with serum $\operatorname{IgG}(\mathrm{rho}=0.6, p<0.01)$ and $\operatorname{IgA}(\mathrm{rho}=0.6, p<0.001$ ), both ASC-derived class-switched antibodies, but not with IgM levels which is produced without class-switching. Moderate correlations were also seen between B-cell activation factors (CD40, CD40L) and sBCMA levels. ${ }^{*} p<0.05,{ }^{* *} p<0.01$, and ${ }^{* * *} p<0.001$.

number of infections recorded within the subsequent 2 years after the initial immunoglobulin measurements. Patients with infections had median IgG, IgA, and IgM levels of $909 \mathrm{mg} / \mathrm{dL}$ (IQR 501), $152 \mathrm{mg} / \mathrm{dl}$ (IQR 177), and $115.5 \mathrm{mg} / \mathrm{dl}$ (IQR 36), respectively. Median serum IgG, IgA, and IgM were $1190 \mathrm{mg} / \mathrm{dl}$ (IQR 390), $210 \mathrm{mg} / \mathrm{dl}$ (IQR 111), and $107 \mathrm{mg} / \mathrm{dl}$ (IQR 71) in the group of RA patients without infections. As shown on Figure 5, there were a few subjects with IgG levels below normal adult ranges $(<639 \mathrm{mg} / \mathrm{dl})$ [33] in both groups and one subject with undetectable IgA levels in the group of RA patients without infections. IgM levels were all within normal adult range concentrations. Importantly, when median levels of the three immunoglobulin isotypes (IgG, IgA, and IgM) were compared between the two RA groups, no statistically significant differences were found (Figure 5).

\section{Discussion}

Rheumatoid arthritis is a disorder of immune dysregulation characterized by enhanced inflammation and increased susceptibility to infections [1,2]. Autoantibody production suggests ASC dysfunction and is central to RA disease pathogenesis $[1,2,35]$. However, how ASC are dysregulated in RA remains inadequately defined. Our study is aimed at studying this phenomenon by examining soluble receptors and immune factors involved in critical steps of ASC generation and survival as a means to evaluate ASC activity in RA.

As most membrane-bound receptors and ligands of the TNF-receptor superfamily (e.g., CD40, CD40L, BAFF, APRIL, TACI, and BCMA) are cleaved from cell surfaces upon binding by their ligands, serum levels of these soluble factors are often interpreted as markers of cell activation $[25,27]$. Most studies investigating factors involved in Bcell/ASC activation and survival in RA describe that they are often upregulated $[25,36-40]$. In keeping with these observations, we identified several patients with soluble levels of the above markers exceeding the measurements seen in healthy subjects in our study. However, a group of RA patients had significantly decreased serum measurements of CD40, CD40L, BCMA, and APRIL, but not of BAFF, TACI, or B-cell homing factors in comparison with 


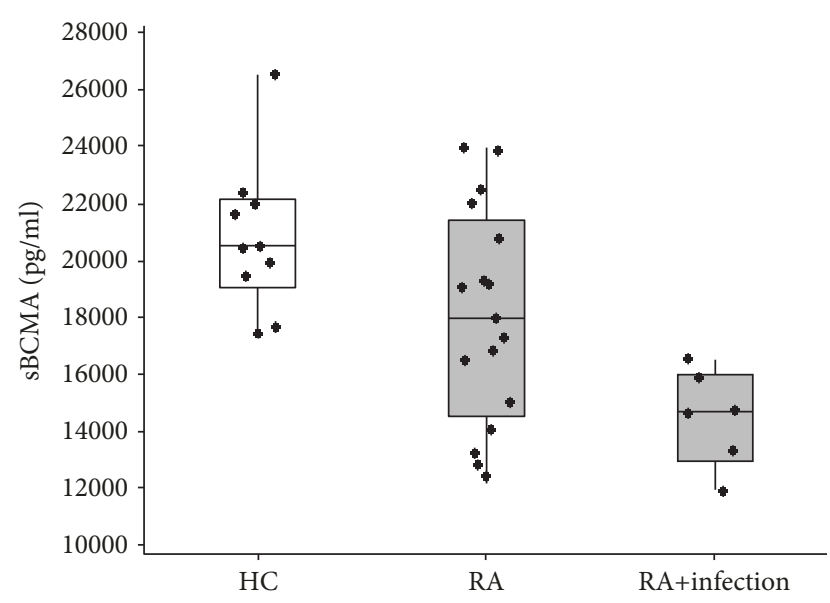

FIGURE 4: Box plots comparing serum sBCMA protein levels among RA patients with and without infections and healthy donors. Levels of sBCMA in healthy controls (HC, left white box; $n=10$ ), RA patients without infections (middle gray box; $n=17$ ), and RA patients with infections (right gray box; $n=6$ ) are shown. Patients with RA with a history of infections had significantly lower sBCMA levels compared to RA subjects without a history of infections $(p<0.05)$ and to healthy controls $(p<0.01) .{ }^{* *} p<0.01$ and ${ }^{*} p<0.05$.

healthy controls. Considering the biological function of these elements, these findings suggested that key steps in mature B-cell activation and/or ASC survival could be impaired in a subset of RA patients.

To determine the clinical significance of the above findings, we next sought to examine the relationship of the above markers of B-cell activation and ASC survival with immunoglobulin production and occurrence of infections, two clinical outcomes related to ASC function [14]. Interestingly, we found that serum sBCMA, but not CD40, CD40L, or APRIL, correlated with serum levels of IgG and IgA, class-switched antibodies, usually produced by long-lived plasma cells [14], but not with serum IgM, which is produced before class-switching, and it is the major antibody of shortlived, primary antibody responses [14]. In turn, decreased serum $\triangle B C M A$ levels were also associated with increased frequency of infections in RA patients. Indeed, all RA patients with an infection in our dataset had serum sBCMA levels below the lowest level observed in healthy subjects and were significantly decreased compared to RA subjects without infectious events. In spite of the small sample, we continued to observe this trend among untreated RA patients showing that low serum sBCMA levels occur even in the absence of medications. Taken together, since sBCMA is released predominantly by ASC, and in its membranebound form, it is the main survival receptor on the surface of long-lived plasma cells [15, 26, 27]; these findings suggested that a paucity in ASC activity and possibly in ASC survival could underlie deficient production of protective antibodies and increased susceptibility to infections in the subgroup of RA patients characterized by lower serum sBCMA.

Defects in ASC function in RA may result from primary disease pathogenesis, secondary to the disease course or to treatments or as a combination of the above. For instance, if low serum sBCMA levels reflect paucity of BCMA at the cell level, ASC survival could be compromised [26]. Alternatively, BCMA could be the result of impaired ASC generation and hence a consequence of decreased cell counts [27]. Specifically, as suggested by the observed decrease in soluble CD40L levels, anomalous CD40L-mediated costimulation by T-helper cells could impair ASC formation and production of IgG and $\operatorname{IgA}[23,41]$. Intrinsic B-cell defects at antigen recognition, signaling through the B-cell receptor, or at B-cell costimulatory checkpoints could result in deficient B-cell activation and ASC generation [14, 21, 42]. The demonstration of decreased sBCMA serum levels in patients with primary antibody deficiencies and inherited defects in these pathways lend support to this notion [43]. Later in the process, failure to complete ASC differentiation can result from dysregulation of transcription factors that preserve $\mathrm{B}$-cell identity (e.g., PAX5, PU.1-IRF-8, and BACH2) [14, 44] or that promote B-cell transformation into ASC (e.g., BLIMP1, XBP1, or IRF4) $[14,44]$. Previous descriptions of RA risk loci on genes involved in the regulation of ASC generation (e.g., IRF4, IRF8, and BLIMP1) [45, 46] support a role for these factors in RA. On the other hand, changes occurring in the natural history of RA or in response to RA treatment could affect ASC activity and impact serum sBCMA release. For instance, cell exhaustion as a consequence of chronic inflammatory disease may blunt B-cell activation and ASC activity $[47,48]$. Likewise, methotrexate, as a folate antagonist, may contribute through impairment of B-cell proliferation [49]. In summary, our findings that serum sBCMA is differentially expressed in a subset of patients with RA prompt multiple considerations of how these changes occur and underscores the need for additional investigation into the mechanisms governing ASC generation and survival as well as secondary events affecting ASC function in RA.

We note that serum immunoglobulin concentrations, including those of IgG and IgA, were not associated with the occurrence of infectious events in patients with RA. A possible explanation for this observation is that autoantibodies represent a constitutive fraction of serum immunoglobulins $[28,50]$ in RA; thus, total IgG and IgA levels may rise if high levels of autoantibodies are present [51]. This idea is supported by our finding that patients with high serum IgG and IgA levels in the setting of low sBCMA had high titers of ACPA antibodies. This observation raises the question as to whether serum immunoglobulin concentrations may not accurately reflect the production of protective antibodies against infections in patients with circulating autoantibodies and additional functional testing of humoral immune responses (e.g., measurements of immunizations responses) and/or additional measurements of ASC activity such as sBCMA should be used to evaluate humoral immunity against infections in RA.

Our study has several limitations. The main caveat of our approach is that the detection of soluble mediators in serum does not necessarily imply that they are functional [25], and additional studies at the cellular level are required to establish the origins of the observed changes. As a crosssectional study, temporal and causal relationships cannot 

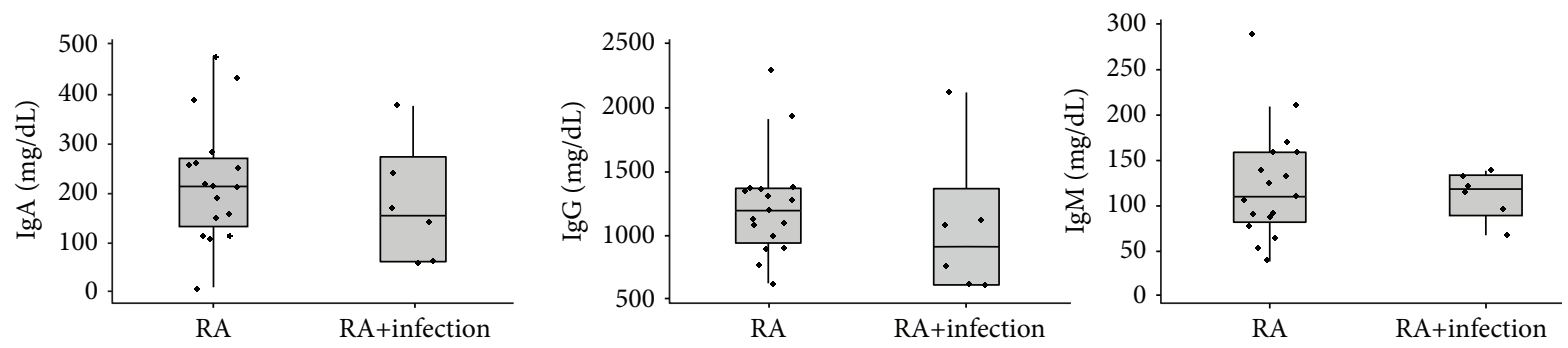

\begin{tabular}{lccc}
\hline Ig isotype $(\mathrm{mg} / \mathrm{dL})$ & RA & RA+infection & $p$-value \\
\hline IgG (median, range) & $1190(610-2290)$ & $909(599-2120)$ & 0.12 \\
IgA (median, range) & $210(4.9-472)$ & $152(54-375)$ & 0.29 \\
IgM (median, range) & $107(37-284)$ & $115.5(64-136)$ & 0.97
\end{tabular}

FIgURE 5: Box plots and analyses comparing serum immunoglobulins (IgG, IgA, or IgM) in RA patients with infections and without infections. The median value is indicated with a horizontal line, with the box comprising the IQR and whiskers representing the maximum and minimum values within the fences $( \pm 1.5 \mathrm{IQR})$. Comparisons between groups were performed using Wilcoxon rank-sum tests. No statistically significant differences were found in serum IgG, IgA, or IgM between the two patient groups.

be established, and our retrospective analysis of cohort data may also be at risk of selection, recall, and attrition bias. Additionally, because of the small sample, our analyses did not include additional factors that modify immune responses in RA such as disease activity or medication use. Nonetheless, we describe significant differences in biologically relevant mediators of B-cell activation (CD40 and CD40L) and ASC survival (sBCMA) and suggest that these findings may be common in RA. Moreover, we have demonstrated an association between a marker of ASC activity, serum sBCMA, and relevant outcomes that are biologically and clinically associated with ASC function such as production of classswitched immunoglobulins and occurrence of infections. These findings prompt further investigation into ASC biology in RA, as these clinically relevant outcomes may relate to anomalies at the cellular level that are susceptible to intervention [25].

\section{Conclusion}

Our study suggests that deficient ASC generation or survival occurs in a subset of RA patients, a novel finding. Elucidating how ASC are regulated in RA may offer novel insights into disease pathogenesis. We establish if soluble immune receptors and factors linked to ASC activity may be used to predict ASC-related clinical events (e.g., infection risk, vaccination responses, and autoantibody production) and inform personalized strategies to modify those outcomes. Furthermore, examining the interplay between ASC function and immunomodulatory drugs may guide a better choice of immunosuppressants and, in the future, provide the basis upon which new preventive and therapeutic approaches are developed.

\section{Data Availability}

The datasets generated and/or analyzed during the current study are available from the corresponding authors on reasonable request.

\section{Ethical Approval}

The Johns Hopkins Institutional Review Board has approved this study, and all participants signed informed consent forms.

\section{Disclosure}

A preliminary version of this work was presented as an abstract at https://www.jacionline.org/article/S00916749(17)32809-9/fulltext.

\section{Conflicts of Interest}

Dr. Desiderio reports consulting fees from Genentech, Merck, and AbbVie. The authors declared that they have no conflict of interest.

\section{Authors' Contributions}

MJG and COB designed the experiments. LC, ED, MJ, and $\mathrm{COB}$ provided clinical data and serum biospecimens. MJG performed data analysis, and SVD, NYW, GN, and COB contributed to the interpretation of results. MJG wrote the manuscript, and all authors revised it for important intellectual content, provided critical feedback, and helped shape the manuscript.

\section{Acknowledgments}

This work was supported by the Cupid Foundation Discovery Award (MJG), the Johns Hopkins School of Medicine Clinician Scientist Award (MJG), and the Jerome L. Greene Foundation (COB) and by Cores B and C of P30AR070254 from the National Institute of Arthritis, Musculoskeletal and Skin Diseases. Dr. Wang receives support from the Johns Hopkins Institute for Clinical and Translational Research funded by grant UL1TR001079 from the National Center for Advancing Translational Science (NCATS). The JHAC 
is maintained with the support of the Camille Julia Morgan Arthritis Research and Education Fund, Joanne and Adam Rogers, and the Scherr Family Fund. Findings on this manuscript including conclusions are entirely of the authors and do not represent the opinions of the funding entities. We thank Tricia Niles and Chloe Kwon from the Becton Dickinson Immune Function Laboratory at the Johns Hopkins Bloomberg School of Public Health, for Luminex support/technical assistance. The facility is supported in part by Centers for Aids Research (CFAR): 5P30AI094189-04 (PI: Dr. Richard Chaisson).

\section{Supplementary Materials}

We are submitting two additional figures as supplementary material to this manuscript. Supplementary Figure 1 displays scatter plots with individual data points of B-cell/ASC soluble markers by age in RA patients and controls. Supplementary Figure 2 displays the comparison of sBCMA levels among controls and untreated RA patients with and without infections. (Supplementary Materials)

\section{References}

[1] J. S. Smolen, D. Aletaha, and I. B. McInnes, "Rheumatoid arthritis," Lancet, vol. 388, no. 10055, pp. 2023-2038, 2016.

[2] G. S. Firestein and I. B. McInnes, "Immunopathogenesis of rheumatoid arthritis," Immunity, vol. 46, no. 2, pp. 183196, 2017.

[3] S. Cobb, F. Anderson, and W. Bauer, "Length of life and cause of death in rheumatoid arthritis," The New England Journal of Medicine, vol. 249, no. 14, pp. 553-556, 1953.

[4] J. Baum, "Infection in rheumatoid arthritis," Arthritis and Rheumatism, vol. 14, no. 1, pp. 135-137, 1971.

[5] W. C. Walker, "Pulmonary infections and rheumatoid arthritis," The Quarterly Journal of Medicine, vol. 36, no. 142, pp. 239-251, 1967.

[6] W. E. Miall, "Rheumatoid arthritis in males; an epidemiological study of a Welsh mining community," Annals of the Rheumatic Diseases, vol. 14, no. 2, pp. 150-158, 1955.

[7] J. Uddin, A. S. S. Kraus Fapha, and H. Garfield Kelly, "Survivorship and death in rheumatoid arthritis," Arthritis and Rheumatism, vol. 13, no. 2, pp. 125-130, 1970.

[8] J. A. Singh, C. Cameron, S. Noorbaloochi et al., "Risk of serious infection in biological treatment of patients with rheumatoid arthritis: a systematic review and meta-analysis," Lancet, vol. 386, no. 9990, pp. 258-265, 2015.

[9] J. F. Ludvigsson, M. Neovius, and L. Hammarstrom, “Association between IgA deficiency \& other autoimmune conditions: a population-based matched cohort study," Journal of Clinical Immunology, vol. 34, no. 4, pp. 444-451, 2014.

[10] S. Kästner, P. Kästner, H.-J. Wolff, and R. Bräuer, “AB0359 deficiencies of Igg and its subclasses in patients suffering from rheumatoid arthritis," Annals of the Rheumatic Diseases, vol. 73, Supplement 2, pp. 923.2-92923, 2014.

[11] A. H. Lee, A. I. Levinson, and H. R. Schumacher Jr., "Hypogammaglobulinemia and rheumatic disease," Seminars in Arthritis and Rheumatism, vol. 22, no. 4, pp. 252-264, 1993.

[12] C. O. Bingham III, R. J. Looney, A. Deodhar et al., "Immunization responses in rheumatoid arthritis patients treated with rituximab: results from a controlled clinical trial," Arthritis and Rheumatism, vol. 62, no. 1, pp. 64-74, 2010.

[13] I. de la Torre, M. J. Leandro, L. Valor, E. Becerra, J. C. W. Edwards, and G. Cambridge, "Total serum immunoglobulin levels in patients with RA after multiple B-cell depletion cycles based on rituximab: relationship with B-cell kinetics," Rheumatology, vol. 51, no. 5, pp. 833-840, 2012.

[14] S. L. Nutt, P. D. Hodgkin, D. M. Tarlinton, and L. M. Corcoran, "The generation of antibody-secreting plasma cells," Nature Reviews. Immunology, vol. 15, no. 3, pp. 160171, 2015.

[15] J. L. Halliley, C. M. Tipton, J. Liesveld et al., "Long-lived plasma cells are contained within the $\mathrm{CD} 19^{-} \mathrm{CD} 38^{\text {hi }} \mathrm{CD} 138^{+}$ subset in human bone marrow," Immunity, vol. 43, no. 1, pp. 132-145, 2015.

[16] S. Iwata and Y. Tanaka, "B-cell subsets, signaling and their roles in secretion of autoantibodies," Lupus, vol. 25, no. 8, pp. 850-856, 2016.

[17] A. N. Theofilopoulos, D. H. Kono, and R. Baccala, "The multiple pathways to autoimmunity," Nature Immunology, vol. 18, no. 7, pp. 716-724, 2017.

[18] M. J. Gutierrez, K. E. Sullivan, R. Fuleihan, USIDNET Consortium, and Bingham CO 3rd, Seminars in Arthritis and Rheumatism, vol. 48, no. 2, 2018Phenotypic characterization of patients with rheumatologic manifestations of common variable immunodeficiency, 2018.

[19] C. Cunningham-Rundles and C. Bodian, "Common variable immunodeficiency: clinical and immunological features of 248 patients," Clinical Immunology, vol. 92, no. 1, pp. 3448, 1999.

[20] P. J. Maglione, "Autoimmune and lymphoproliferative complications of common variable immunodeficiency," Current Allergy and Asthma Reports, vol. 16, no. 3, p. 19, 2016.

[21] V. T. Chu and C. Berek, "The establishment of the plasma cell survival niche in the bone marrow," Immunological Reviews, vol. 251, no. 1, pp. 177-188, 2013.

[22] N. S. De Silva and U. Klein, "Dynamics of B cells in germinal centres," Nature Reviews Immunology, vol. 15, no. 3, pp. 137-148, 2015.

[23] I. S. Grewal and R. A. Flavell, "The role of CD40 ligand in costimulation and T-cell activation," Immunological Reviews, vol. 153, no. 1, pp. 85-106, 1996.

[24] C. Bossen and P. Schneider, "BAFF, APRIL and their receptors: structure, function and signaling," Seminars in Immunology, vol. 18, no. 5, pp. 263-275, 2006.

[25] M. Croft and R. M. Siegel, "Beyond TNF: TNF superfamily cytokines as targets for the treatment of rheumatic diseases," Nature Reviews Rheumatology, vol. 13, no. 4, pp. 217233, 2017.

[26] B. P. O'Connor, V. S. Raman, L. D. Erickson et al., "BCMA is essential for the survival of long-lived bone marrow plasma cells," The Journal of Experimental Medicine, vol. 199, no. 1, pp. 91-98, 2004.

[27] S. A. Laurent, F. S. Hoffmann, P. H. Kuhn et al., " $\gamma$-Secretase directly sheds the survival receptor BCMA from plasma cells," Nature Communications, vol. 6, no. 1, article 7333, 2015.

[28] S. Procaccia, A. Gasparini, A. Colucci et al., "ELISA determined IgM, IgG and IgA rheumatoid factors in rheumatoid arthritis and in other connective tissue diseases," Clinical and Experimental Rheumatology, vol. 5, no. 4, pp. 335342, 1987. 
[29] S. Bugatti, B. Vitolo, R. Caporali, C. Montecucco, and A. Manzo, "B cells in rheumatoid arthritis: from pathogenic players to disease biomarkers," BioMed Research International, vol. 2014, Article ID 681678, 14 pages, 2014.

[30] L. C. Cappelli, M. F. Konig, A. C. Gelber, C. O. Bingham, and E. Darrah, "Smoking is not linked to the development of anti-peptidylarginine deiminase 4 autoantibodies in rheumatoid arthritis," Arthritis Research \& Therapy, vol. 20, no. 1, p. 59, 2018.

[31] D. Aletaha, T. Neogi, A. J. Silman et al., "2010 rheumatoid arthritis classification criteria: an American College of Rheumatology/European League Against Rheumatism collaborative initiative," Arthritis and Rheumatism, vol. 62, no. 9, pp. 2569-2581, 2010.

[32] D. Aletaha, V. P. K. Nell, T. Stamm et al., "Acute phase reactants add little to composite disease activity indices for rheumatoid arthritis: validation of a clinical activity score," Arthritis Research \& Therapy, vol. 7, no. 4, pp. R796R806, 2005.

[33] C. R. Jolliff, K. M. Cost, P. C. Stivrins et al., "Reference intervals for serum IgG, IgA, IgM, C3, and C4 as determined by rate nephelometry," Clinical Chemistry, vol. 28, no. 1, pp. 126$128,1982$.

[34] J. B. Oliveira and T. A. Fleisher, "Laboratory evaluation of primary immunodeficiencies," Journal of Allergy and Clinical Immunology, vol. 125, no. 2, pp. S297-S305, 2010.

[35] A. Dahdah, K. Habir, K. S. Nandakumar et al., "Germinal center B cells are essential for collagen-induced arthritis," Arthritis \& Rhematology, vol. 70, no. 2, pp. 193-203, 2018.

[36] J. E. Gottenberg, C. Miceli-Richard, B. Ducot, P. Goupille, B. Combe, and X. Mariette, "Markers of B-lymphocyte activation are elevated in patients with early rheumatoid arthritis and correlated with disease activity in the ESPOIR cohort," Arthritis Research \& Therapy, vol. 11, no. 4, article R114, 2009.

[37] K. Nakajima, K. Itoh, K. Nagatani et al., "Expression of BAFF and BAFF-R in the synovial tissue of patients with rheumatoid arthritis," Scandinavian Journal of Rheumatology, vol. 36, no. 5, pp. 365-372, 2009.

[38] T. M. Seyler, Y. W. Park, S. Takemura et al., "BLyS and APRIL in rheumatoid arthritis," Journal of Clinical Investigation, vol. 115, no. 11, pp. 3083-3092, 2005.

[39] A. L. Peters, L. L. Stunz, and G. A. Bishop, "CD40 and autoimmunity: the dark side of a great activator," Seminars in Immunology, vol. 21, no. 5, pp. 293-300, 2009.

[40] J. Zhao, J. Guo, L. Wang, W. Zhou, and Z. Zhang, "The role of a proliferation-inducing ligand (APRIL) in the pathogenesis of rheumatoid arthritis," Scandinavian Journal of Rheumatology, vol. 43, no. 6, pp. 462-469, 2014.

[41] R. C. Allen, R. J. Armitage, M. E. Conley et al., "CD40 ligand gene defects responsible for X-linked hyper-IgM syndrome," Science, vol. 259, no. 5097, pp. 990-993, 1993.

[42] S. Crotty, "A brief history of T cell help to B cells," Nature Reviews Immunology, vol. 15, no. 3, pp. 185-189, 2015.

[43] P. J. Maglione, M. Li, E. Sanchez et al., "Reduced serum BCMA levels distinguish patients with primary antibody deficiency," The Journal of Immunology, vol. 196, Supplement 1, p. 193.7, 2016.

[44] W. Shi, Y. Liao, S. N. Willis et al., "Transcriptional profiling of mouse B cell terminal differentiation defines a signature for antibody-secreting plasma cells," Nature Immunology, vol. 16 , no. 6 , pp. 663-673, 2015.
[45] the RACI consortium, the GARNET consortium, Y. Okada et al., "Genetics of rheumatoid arthritis contributes to biology and drug discovery," Nature, vol. 506, no. 7488, pp. 376381, 2014.

[46] S. J. Kim, "Immunological function of Blimp-1 in dendritic cells and relevance to autoimmune diseases," Immunologic Research, vol. 63, no. 1-3, pp. 113-120, 2015.

[47] L. Yeo, H. Lom, M. Juarez et al., "Expression of FcRL4 defines a pro-inflammatory, RANKL-producing B cell subset in rheumatoid arthritis," Annals of the Rheumatic Diseases, vol. 74, no. 5, pp. 928-935, 2015.

[48] S. Moir and A. S. Fauci, "B-cell exhaustion in HIV infection: the role of immune activation," Current Opinion in HIV and AIDS, vol. 9, no. 5, pp. 472-477, 2014.

[49] P. M. Brown, A. G. Pratt, and J. D. Isaacs, "Mechanism of action of methotrexate in rheumatoid arthritis, and the search for biomarkers," Nature Reviews Rheumatology, vol. 12, no. 12, pp. 731-742, 2016.

[50] E. P. Nagele, M. Han, N. K. Acharya, C. DeMarshall, M. C. Kosciuk, and R. G. Nagele, "Natural IgG autoantibodies are abundant and ubiquitous in human sera, and their number is influenced by age, gender, and disease," PLoS One, vol. 8, no. 4, article e60726, 2013.

[51] J. Barden, F. Mullinax, and M. Waller, "Immunoglobulin levels in rheumatoid arthritis: comparison with rheumatoid factor titers, clinical stage and disease duration," Arthritis and Rheumatism, vol. 10, no. 3, pp. 228-234, 1967. 


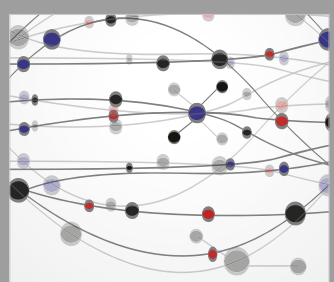

The Scientific World Journal
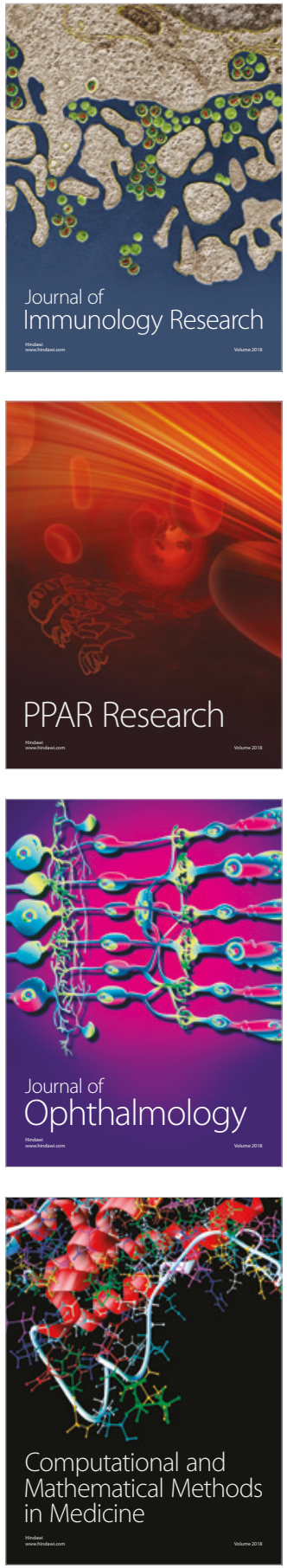

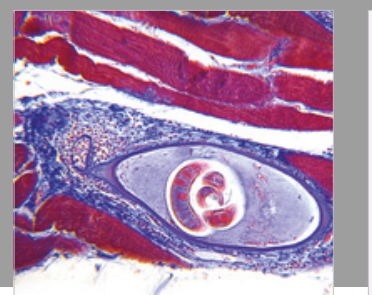

Gastroenterology Research and Practice

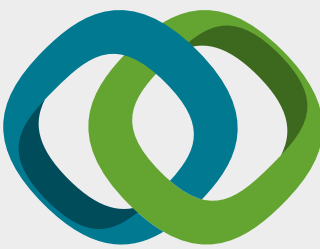

\section{Hindawi}

Submit your manuscripts at

www.hindawi.com
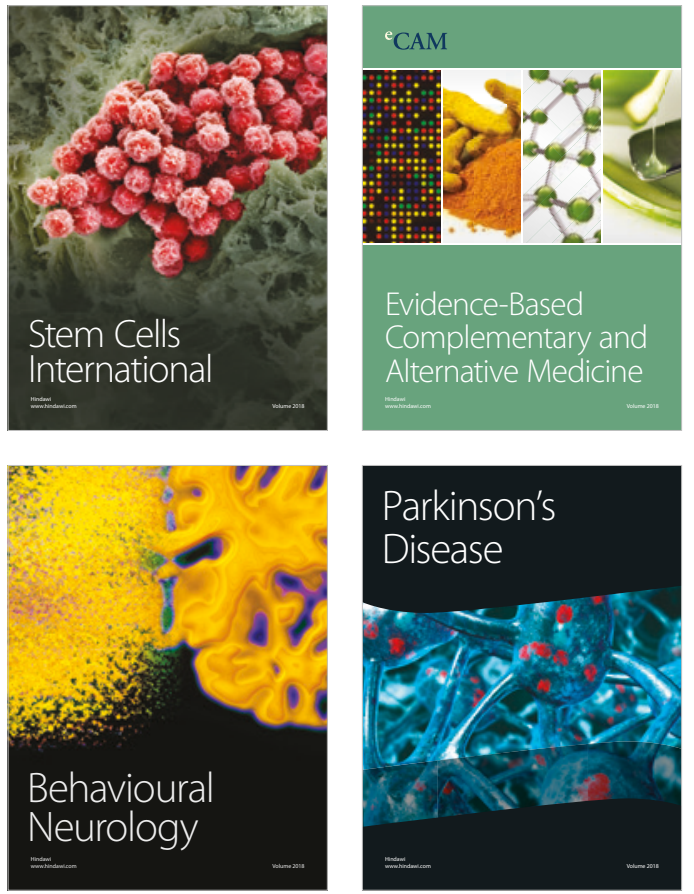

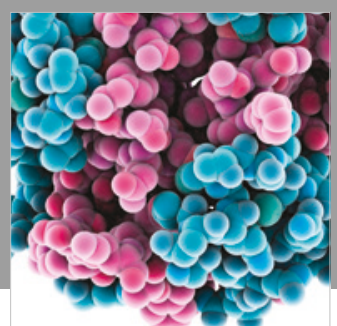

ournal of

Diabetes Research

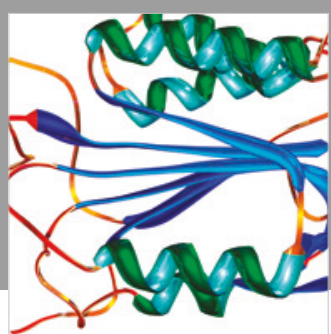

Disease Markers
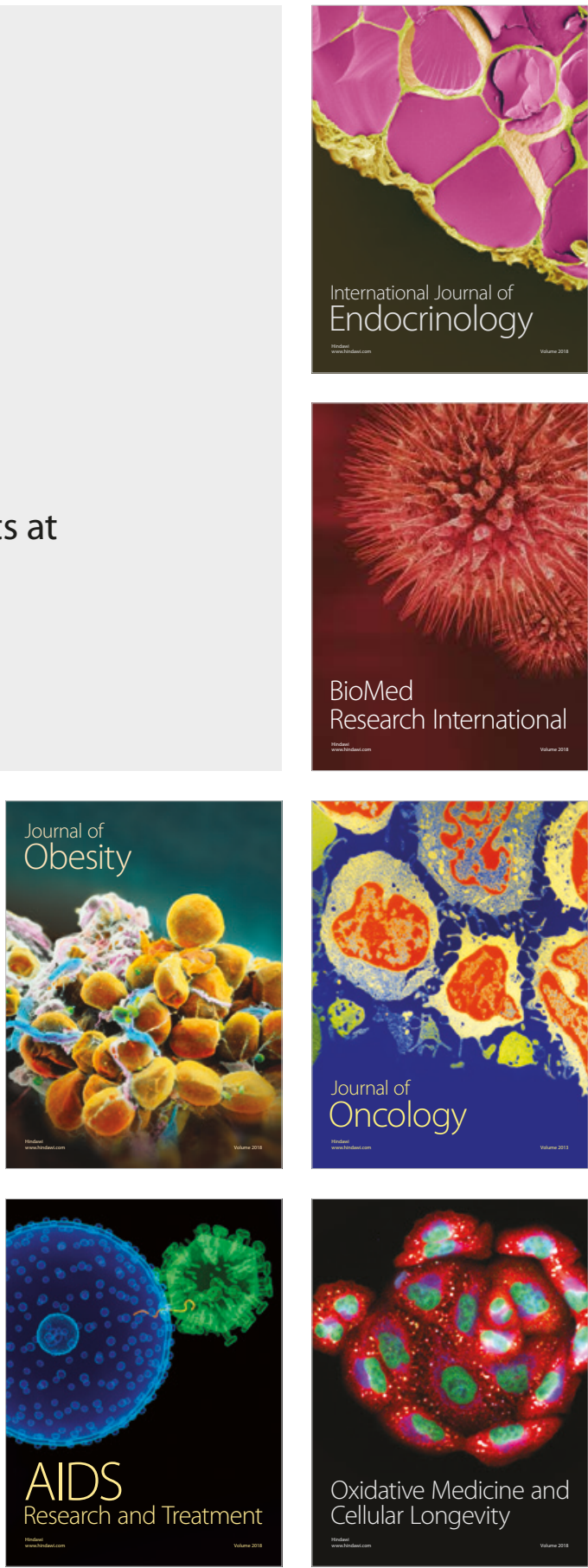